\title{
DECAY BOUNDS FOR NONLOCAL EVOLUTION EQUATIONS IN ORLICZ SPACES
}

\author{
URIEL KAUFMANN, JULIO D. ROSSI AND RAUL VIDAL
}

Abstract. We show decay bounds of the form

$$
\int_{\mathbb{R}^{d}} \phi(u(x, t)) d x \leq C t^{-\mu}
$$

for integrable and bounded solutions to the nonlocal evolution equation

$$
u_{t}(x, t)=\int_{\mathbb{R}^{d}} J(x, y) G(u(y, t)-u(x, t))(u(y, t)-u(x, t)) d y+f(u(x, t)) .
$$

Here $G$ is a nonnegative and even function and $f$ verifies $f(\xi) \xi \leq 0$ for all $\xi \geq 0$. We remark that $G$ is not assumed to be homogeneous. The function $\phi$ and the exponent $\mu$ depend on $G$ via adequate hypotheses, while $J$ is a nonnegative kernel satisfying suitable assumptions.

\section{INTRODUCTION}

In this paper we deal with the long-time behavior of bounded solutions to the equation

$$
u_{t}(x, t)=\int_{\mathbb{R}^{d}} J(x, y) G(u(y, t)-u(x, t))(u(y, t)-u(x, t)) d y+f(u(x, t)),
$$

with $(x, t) \in \mathbb{R}^{d} \times(0, \infty)$ and a nonnegative initial condition

$$
u(\cdot, 0)=u_{0}(\cdot) \in L^{1}\left(\mathbb{R}^{d}\right) \cap L^{\infty}\left(\mathbb{R}^{d}\right) .
$$

Here $J$ is a nonnegative symmetric and smooth kernel satisfying some suitable assumptions (see Lemma 2.2 below) and $G: \mathbb{R} \rightarrow[0, \infty)$ is an even function. The nonlinearity $f$ is assumed to verify that $f(\xi) \xi \leq 0$ for all $\xi \geq 0$.

Under these conditions it can be proved as in [1] that problem (1.1) has a unique solution that is nonnegative, integrable and bounded. In fact, we have that

$$
\|u(\cdot, t)\|_{L^{\infty}\left(\mathbb{R}^{d}\right)} \leq\|u(\cdot, 0)\|_{L^{\infty}\left(\mathbb{R}^{d}\right)}:=M>0 .
$$

Now we introduce the following condition: Suppose there exist a $C^{1}$ Young function $\phi: \mathbb{R} \rightarrow[0, \infty)$, and an even function $\theta: \mathbb{R} \rightarrow[0, \infty)$, such that

$$
G(\xi-\eta)(\xi-\eta)\left(\phi^{\prime}(\xi)-\phi^{\prime}(\eta)\right) \geq \theta(\xi-\eta)
$$

for all $0 \leq \xi, \eta \leq M$.

Key words and phrases. Nonlocal diffusion, energy methods, Orlicz spaces.

2010 Mathematics Subject Classification: 47G10, 47J35, 45G10. 
We remark that we are not assuming that $G$ is homogeneous of any kind (as happens, for example, for $\left.G(\xi)=|\xi|^{p-2}\right)$.

We will prove in our main result (see Theorem 3.2) that for all $t$ sufficiently large the solution $u$ of (1.1) satisfies the decay estimate

$$
\int_{\mathbb{R}^{d}} \phi(u(x, t)) d x \leq C t^{-\mu},
$$

where $\mu>0$ is given explicitly and depends on $\phi, \theta$ and $d$. As a consequence of this result we are also able to provide decay bounds for the Orlicz norm of the solutions (see Corollary 3.6).

Let us end this introduction with a brief description of the ideas used in the proofs and the connection with previous results. To study the decay of solutions to nonlocal nonlinear evolution equations like (1.1) we will use energy methods as the ones introduced in [12]. Note that the kernel $J$ is smooth (therefore we don't have a regularizing effect, see [1]) and not in convolution form (hence Fourier methods like the ones used in [5] can not be applied). Let us mention that nonlocal problems analogous to the $p$-Laplacian (i.e., with $G(\xi)=|\xi|^{p-2}$ ) have already been studied in the literature. In [2] and [3] they were considered under the assumption that $J$ is a convolution kernel, while in Theorem 4.2 in [12] decay estimates similar to those in Theorem 3.2 where obtained in the case $p \in[2, d)$ and $f \equiv 0$. Let us note that the bounds stated here for the particular case $G(\xi)=|\xi|^{p-2}$ do not impose any restriction on $p$ (see Remark 3.3 below). Nonlocal problems have been recently widely used to model diffusion processes (see [9] and [8] for a general nonlocal vector calculus) and are closely connected with real applications. We mention, for example, peridynamics, a recent nonlocal model for elasticity. For extra references concerning applications we refer for instance to [4], [11], [14], [15], [16] and the recent book [1].

Concerning singular kernels (that are related to fractional type operators) we quote [6], [7] and references therein.

The rest of the paper is organized as follows: in Section 2 we collect some preliminary results including a key lemma (Lemma 2.2) that provides a decomposition of a function in a smooth and a rough part whose norms are controlled by a double integral involving $J$ and $|u(x)-u(y)|^{p}$ (it is here where we need to assume extra conditions on $J$ ); finally in Section 3 we prove our main result, Theorem 3.2, where we show the bound (1.4) for an explicit $\mu$ that depends on suitable conditions that we assume for the functions $\theta$ and $\phi$ that appear in (1.3).

\section{Preliminary Results}

As usual, we say that $\phi: \mathbb{R} \rightarrow[0, \infty)$ is a Young function if $\phi$ is convex and even, $\phi(0)=0$ and $\lim _{\xi \rightarrow \infty} \phi(\xi)=\infty$. Following [10], we say that a Young function $\phi$ is of upper type $p$, for some $p>1$, if there exists a constant $C_{\phi}>0$ such that

$$
\phi(\lambda \xi) \leq C_{\phi} \lambda^{p} \phi(\xi)
$$

for every $\lambda>1$ and every $\xi \geq 0$. We also set $\phi^{-1}(\xi):=\inf \{\eta: \phi(\eta)>\xi\}$. 
We will need the following lemma that gives a sort of interpolation inequality.

Lemma 2.1. Let $\phi, \phi_{*}$ be two Young functions such that for all $\xi \geq 0$

$$
\phi^{-1}(\xi) \geq C \xi^{\lambda}\left(\phi_{*}^{-1}(\xi)\right)^{1-\lambda}
$$

for some $\lambda \in(0,1)$ and $C>0$. Then for all $f \in L^{1}\left(\mathbb{R}^{d}\right) \cap L^{\phi_{*}}\left(\mathbb{R}^{d}\right)$ it holds that

$$
\|f\|_{L^{\phi}\left(\mathbb{R}^{d}\right)} \leq \frac{2}{C}\|f\|_{L^{1}\left(\mathbb{R}^{d}\right)}^{\lambda}\|f\|_{L^{\phi_{*}\left(\mathbb{R}^{d}\right)}}^{1-\lambda} .
$$

Proof. Let

$$
\psi(\xi):=\xi^{1 /(1-\lambda)} \quad \text { and } \quad \varphi(\xi):=(\xi / C)^{1 / \lambda} .
$$

We observe that

$$
\begin{gathered}
\left\||f|^{1-\lambda}\right\|_{L^{\phi_{*} \alpha \psi}\left(\mathbb{R}^{d}\right)}=\inf \left\{\lambda>0: \int_{\mathbb{R}^{d}} \phi_{*}\left(\psi\left(\frac{|f|^{1-\lambda}}{\lambda}\right)\right) \leq 1\right\} \\
=\inf \left\{\lambda>0: \int_{\mathbb{R}^{d}} \phi_{*}\left(\frac{|f|}{\psi(\lambda)}\right) \leq 1\right\} \\
=\inf \left\{\mu^{1-\lambda}>0: \int_{\mathbb{R}^{d}} \phi_{*}\left(\frac{|f|}{\mu}\right) \leq 1\right\}=\|f\|_{L^{\phi_{*}\left(\mathbb{R}^{d}\right)}}^{1-\lambda},
\end{gathered}
$$

and it is easy to check that

$$
\left\||f|^{\lambda}\right\|_{L^{\varphi}\left(\mathbb{R}^{d}\right)}=\|f\|_{L^{1}\left(\mathbb{R}^{d}\right)}^{\lambda} / C .
$$

Note also that (2.1) says that

$$
\varphi^{-1}(\xi)\left(\phi_{*} \circ \psi\right)^{-1}(\xi) \leq \phi^{-1}(\xi) .
$$

Therefore, from the above mentioned facts and Hölder's inequality (e.g. [13], Theorem 2.3) we get that

$$
\begin{aligned}
\|f\|_{L^{\phi}\left(\mathbb{R}^{d}\right)} & =\left\||f|^{\lambda}|f|^{1-\lambda}\right\|_{L^{\phi}\left(\mathbb{R}^{d}\right)} \\
& \leq 2\left\||f|^{\lambda}\right\|_{L^{\varphi}\left(\mathbb{R}^{d}\right)}\left\||f|^{1-\lambda}\right\|_{L^{\phi_{*} \circ \psi}\left(\mathbb{R}^{d}\right)} \\
& =\frac{2}{C}\|f\|_{L^{1}\left(\mathbb{R}^{d}\right)}^{\lambda}\|f\|_{L^{\phi_{*}}\left(\mathbb{R}^{d}\right)}^{1-\lambda} .
\end{aligned}
$$

This concludes the proof.

We state for the sake of completeness the following decomposition result (for the proof we refer to Theorem 2.1 in [12]).

Lemma 2.2. Let $p \in[1, \infty)$ and let $J(\cdot, \cdot): \mathbb{R}^{d} \times \mathbb{R}^{d} \rightarrow \mathbb{R}$ be a symmetric nonnegative function such that

HJ1) There exists $0<C<\infty$ such that

$$
\sup _{y \in \mathbb{R}^{d}} \int_{\mathbb{R}^{d}} J(x, y) d x \leq C .
$$

HJ2) There exist $c_{1}, c_{2}>0$ and a function $a \in C^{1}\left(\mathbb{R}^{d}, \mathbb{R}^{d}\right)$ satisfying

$$
\sup _{\substack{x \in \mathbb{R}^{d} \\ 1 \leq i, j \leq d}}\left|\partial_{x_{i}} a_{j}(x)\right|<\infty,
$$


and

$$
\left\{y \in \mathbb{R}^{d}:|y-a(x)| \leq c_{2}\right\} \subset\left\{y \in \mathbb{R}^{d}: J(x, y)>c_{1}\right\} .
$$

Then, for any $u \in L^{p}\left(\mathbb{R}^{d}\right)$ there exist two functions $v$ and $w$ such that

$$
u=v+w
$$

and

$$
\|\nabla v\|_{L^{p}\left(\mathbb{R}^{d}\right)}^{p}+\|w\|_{L^{p}\left(\mathbb{R}^{d}\right)}^{p} \leq C(J, p) \int_{\mathbb{R}^{d}} \int_{\mathbb{R}^{d}} J(x, y)|u(x)-u(y)|^{p} d y d x
$$

\section{Proof OF THE DECAY BOUNDS.}

First, let us show that $\int_{\mathbb{R}^{d}} \phi(u(x, t)) d x$ is decreasing in time.

Lemma 3.1. Let $J(\cdot, \cdot): \mathbb{R}^{d} \times \mathbb{R}^{d} \rightarrow \mathbb{R}$ be a symmetric nonnegative function, let $\phi$ be a $C^{1}$ Young function, and assume that $G: \mathbb{R} \rightarrow[0, \infty)$ is even and such that there exist $\theta: \mathbb{R} \rightarrow[0, \infty)$ even and $M>0$ such that

$$
G(\xi-\eta)(\xi-\eta)\left(\phi^{\prime}(\xi)-\phi^{\prime}(\eta)\right) \geq \theta(\xi-\eta)
$$

for all $0 \leq \xi, \eta \leq M$. Suppose $f$ is such that $f(\xi) \xi \leq 0$ for all $\xi \geq 0$. If $u$ is the solution of (1.1) with a nonnegative initial datum $u(\cdot, 0)$ with $\|u(\cdot, 0)\|_{L^{\infty}\left(\mathbb{R}^{d}\right)} \leq M$, then

where

$$
\frac{d}{d t} \int_{\mathbb{R}^{d}} \phi(u(x, t)) d x+\frac{1}{2}\left\langle A_{\theta} u, u\right\rangle \leq 0,
$$

$$
\left\langle A_{\theta} u, u\right\rangle:=\int_{\mathbb{R}^{d}} \int_{\mathbb{R}^{d}} J(x, y) \theta(u(y, t)-u(x, t)) d y d x
$$

Proof. We observe first that the assumption $f(\xi) \xi \leq 0$ for every $\xi \geq 0$ implies that $f(\xi) \phi^{\prime}(\xi) \leq 0$ for every $\xi \geq 0$. Indeed, if $\xi \geq 0, \phi^{\prime}(\xi) \geq 0$ and hence $s g\left(f(\xi) \phi^{\prime}(\xi)\right)=s g(f(\xi) \xi)$.

Now let us now compute the following derivative with respect to $t$ :

$$
\begin{aligned}
& \frac{d}{d t} \int_{\mathbb{R}^{d}} \phi(u(x, t)) d x=\int_{\mathbb{R}^{d}} \phi^{\prime}(u(x, t)) u_{t}(x, t) d x \\
& =\int_{\mathbb{R}^{d}} \phi^{\prime}(u(x, t)) \int_{\mathbb{R}^{d}} J(x, y) G(u(y, t)-u(x, t))(u(y, t)-u(x, t)) d y d x \\
& \quad+\int_{\mathbb{R}^{d}} f(u(x, t)) \phi^{\prime}(u(x, t)) d x \\
& \leq \int_{\mathbb{R}^{d}} \int_{\mathbb{R}^{d}} \phi^{\prime}(u(x, t)) J(x, y) G(u(y, t)-u(x, t))(u(y, t)-u(x, t)) d y d x \\
& =-\frac{1}{2} \int_{\mathbb{R}^{d}} \int_{\mathbb{R}^{d}} J(x, y) G(u(y, t)-u(x, t))(u(y, t)-u(x, t)) \\
& \quad \times\left(\phi^{\prime}(u(y, t))-\phi^{\prime}(u(x, t))\right) d y d x \\
& \leq-\frac{1}{2} \int_{\mathbb{R}^{d}} \int_{\mathbb{R}^{d}} J(x, y) \theta(u(y, t)-u(x, t)) d y d x,
\end{aligned}
$$


where the last equality follows as in Lemma 2.1 in [12], and where in order to get the last inequality we have used (3.1). Hence, we have obtained the desired conclusion.

Theorem 3.2. Let $J, G$ and $f$ be as in Lemma 3.1, and let $u$ be the solution of (1.1) with a nonnegative initial datum $u(\cdot, 0)$ such that $\|u(\cdot, 0)\|_{L^{\infty}\left(\mathbb{R}^{d}\right)} \leq M$. Suppose there exist $k_{1}, k_{2}>0$ such that

$$
\theta(\xi) \geq k_{1} \xi^{p} \quad \text { and } \quad \phi(\xi) \leq k_{2} \xi^{q}
$$

for all $0 \leq \xi \leq M$ and some $1<q \leq p$. Then

$$
\int_{\mathbb{R}^{d}} \phi(u(x, t)) d x \leq C t^{-\mu}
$$

for all t sufficiently large, where

$$
\mu:=\frac{d(q-1)}{d(p-q)+p}
$$

Proof. Along this proof we denote by $C$ a constant that may change from one line to another but that is independent of the relevant quantities.

Let $\alpha \in(0,1)$ and $\beta \in(0,1]$ be given by

$$
\frac{1}{p}=\frac{\alpha}{p^{*}}+1-\alpha, \quad \frac{1}{q}=\frac{\beta}{p}+1-\beta,
$$

where as usual

$$
p^{*}=\frac{d p}{d-p} \quad \text { if } p<d
$$

and

$$
p^{*}=+\infty \quad \text { if } p>d \text {. }
$$

Note that

$$
\begin{array}{ll}
\alpha=\frac{1}{p^{\prime}} \frac{p^{*}}{p^{*}-1}=\frac{d(p-1)}{d(p-1)+p} & \text { if } p<d, \\
\alpha=\frac{1}{p^{\prime}} & \text { if } p>d, \\
\beta=\frac{p}{q^{\prime}(p-1)}=\frac{q-1}{q} \frac{p}{p-1} . &
\end{array}
$$

Let $\left\langle A_{p} u, u\right\rangle$ be given by

$$
\left\langle A_{p} u, u\right\rangle:=\int_{\mathbb{R}^{d}} \int_{\mathbb{R}^{d}} J(x, y)|u(y, t)-u(x, t)|^{p} d y d x,
$$

that is, (3.2) with $\theta(\xi)=|\xi|^{p}$.

Now, let us write

$$
u:=v+w,
$$


with $v$ and $w$ as in the decomposition lemma, Lemma 2.2. Interpolating two times, using the aforementioned lemma and Lemma 3.1 we deduce that

$$
\begin{aligned}
\left(k_{2}^{-1}\right. & \left.\int_{\mathbb{R}^{d}} \phi(u)\right)^{1 / q} \leq\|u\|_{L^{q}\left(\mathbb{R}^{d}\right)} \leq\|u\|_{L^{1}\left(\mathbb{R}^{d}\right)}^{1-\beta}\|u\|_{L^{p}\left(\mathbb{R}^{d}\right)}^{\beta} \\
& \leq C\|u\|_{L^{p}\left(\mathbb{R}^{d}\right)}^{\beta}=C\|v+w\|_{L^{p}\left(\mathbb{R}^{d}\right)}^{\beta} \\
& \leq C\left(\|v\|_{L^{p}\left(\mathbb{R}^{d}\right)}+\|w\|_{L^{p}\left(\mathbb{R}^{d}\right)}\right) \\
& \leq C\left(\|v\|_{L^{p}\left(\mathbb{R}^{d}\right)}^{\beta}+\|w\|_{L^{p}\left(\mathbb{R}^{d}\right)}^{\beta}\right) \\
& \leq C\left(\|v\|_{L^{1}\left(\mathbb{R}^{d}\right)}^{\beta(-\alpha)}\|v\|_{L^{p^{*}\left(\mathbb{R}^{d}\right)}}^{\beta \alpha}+\|w\|_{L^{p}\left(\mathbb{R}^{d}\right)}^{\beta}\right) \\
& \leq C\left(\|v\|_{L^{p^{*}\left(\mathbb{R}^{d}\right)}}^{\beta \alpha}+\|w\|_{L^{p}\left(\mathbb{R}^{d}\right)}^{\beta}\right) \\
& \leq C\left(\|\nabla v\|_{L^{p}\left(\mathbb{R}^{d}\right)}^{\beta \alpha}+\|w\|_{L^{p}\left(\mathbb{R}^{d}\right)}^{\beta}\right) \\
& \leq C\left(\left\langle A_{p} u, u\right\rangle^{\beta \alpha / p}+\left\langle A_{p} u, u\right\rangle^{\beta / p}\right) \\
& \leq C\left(\left\langle A_{\theta} u, u\right\rangle^{\beta \alpha / p}+\left\langle A_{\theta} u, u\right\rangle^{\beta / p}\right) \\
& \leq-C\left(\left[\frac{d}{d t}\left(\int_{\mathbb{R}^{d}} \phi(u)\right)\right]^{\beta \alpha / p}-\left[\frac{d}{d t}\left(\int_{\mathbb{R}^{d}} \phi(u)\right)\right]^{\beta / p}\right) .
\end{aligned}
$$

We now set

$$
X(t):=\int_{\mathbb{R}^{d}} \phi(u(x, t)) d x .
$$

From our previous computations we get

$$
\begin{aligned}
X(t) & \leq-C\left(\left[X^{\prime}(t)\right]^{\beta \alpha / p}+\left[X^{\prime}(t)\right]^{\beta / p}\right)^{q} \\
& \leq-C\left(\left[X^{\prime}(t)\right]^{\beta \alpha q / p}+\left[X^{\prime}(t)\right]^{\beta q / p}\right) .
\end{aligned}
$$

If for $0 \leq \xi$ we define $H(\xi):=C\left(\xi^{\beta \alpha q / p}+\xi^{\beta q / p}\right)$ then we have

$$
X^{\prime}(t) \leq-H^{-1}(X(t)) .
$$

We observe next that if $\xi \leq K=\int_{\mathbb{R}^{d}} \phi(u(x, 0)) d x$ there exists $C=C(K)>0$ such that $H^{-1}(\xi) \geq C \xi^{\gamma}$ with $\gamma:=p /(\beta \alpha q)$. Then

$$
X^{\prime}(t) \leq-C\left(X(t)^{\gamma}\right)
$$

and thus integrating we get

$$
X(t) \leq C t^{-1 /(\gamma-1)}
$$

and this ends the proof of the theorem.

Remark 3.3. For the case of pure powers our results read as follows: Let

$$
G(\xi):=|\xi|^{p-2}
$$


for some $p>1$. We choose $\phi(\xi):=|\xi|^{q}$ with $q \in(1,2]$, and $\theta(\xi):=\frac{q(q-1)}{M^{2-q}}|\xi|^{p}$. Suppose $0 \leq \eta \leq \xi \leq M$ (the case $0 \leq \xi \leq \eta \leq M$ is analogous since $G$ and $\theta$ are even). We have that

$$
\begin{aligned}
G(\xi-\eta) & (\xi-\eta)\left(\phi^{\prime}(\xi)-\phi^{\prime}(\eta)\right) \\
& =q(\xi-\eta)^{p} \frac{\left(\xi^{q-1}-\eta^{q-1}\right)}{\xi-\eta} \\
& \geq \frac{q(q-1)}{M^{2-q}}(\xi-\eta)^{p}=\theta(\xi-\eta)
\end{aligned}
$$

and hence (3.1) holds. Let us observe that we impose no condition on $p$. Indeed, in the statement of Theorem 3.2 we only need $p \geq q>1$ and hence given any $p>1$ we can always pick $q \in(1, \min (2, p)]$. Thus, our results extend the ones in [12] where similar bounds where obtained for $f \equiv 0$ and $G(\xi)=|\xi|^{p-2}$ with $p \in[2, d)$.

Remark 3.4. As an example of functions $G$ verifying our conditions that are not necessarily homogeneous we mention that we may consider any even function $G$ satisfying

$$
G(\xi) \geq k \xi^{p-2}
$$

for $\xi \geq 0$ and some $k>0$ and $p>1$. For instance, let

$$
G(\xi):=|\xi|^{p-2} h(\xi)
$$

where $h: \mathbb{R} \rightarrow[0, \infty)$ is an even function with $\inf _{\xi \geq 0} h(\xi)>0$. Indeed, given such a $G$, we take

$$
\phi(\xi):=|\xi|^{q}, \quad \theta(\xi):=k \frac{q(q-1)}{M^{2-q}}|\xi|^{p}
$$

with $q \in(1, \min (2, p)]$. Then, clearly the assumptions for $\phi$ and $\theta$ in Theorem 3.2 are satisfied and one can also check that (3.1) holds arguing as in the previous remark.

Remark 3.5. Let us note that if in addition to the hypotheses of Theorem 3.2 it also holds that

$$
\phi(\xi) \geq k \xi^{r}
$$

for all $0 \leq \xi \leq M$ with $r \geq q$, from (3.3) we get that

$$
\|u\|_{L^{r}\left(\mathbb{R}^{d}\right)} \leq C t^{-\mu / r} \quad \text { for all } t \text { sufficiently large. }
$$

The previous results also provide decay estimates for the Orlicz norm of the solutions of (1.1).

Corollary 3.6. Let $J, G$ and $f$ be as in Theorem 3.2, and let $u$ be the solution to (1.1) with a nonnegative initial datum $u(\cdot, 0)$ such that $\|u(\cdot, 0)\|_{L^{\infty}\left(\mathbb{R}^{d}\right)} \leq M$.

(i) If

$$
c_{1} \xi^{r} \leq \phi(\xi) \leq c_{2} \xi^{\frac{r}{\lambda(r-1)+1}}
$$

for all $0 \leq \xi \leq M$ and some $r \geq q$ and $\lambda \in(0,1)$, then

$$
\|u\|_{L^{\phi}\left(\mathbb{R}^{d}\right)} \leq C t^{-\mu(1-\lambda) / r} \quad \text { for all } t \text { sufficiently large. }
$$

(ii) If $\phi$ is of upper type $s$, then

$$
\|u\|_{L^{\phi}\left(\mathbb{R}^{d}\right)} \leq C t^{-\mu / s} \quad \text { for all } t \text { sufficiently large. }
$$


Proof. Let $\xi \geq 0$. In order to prove (i) we take $\phi_{*}(\xi):=\xi^{r}$ and we note that $\phi(\xi) \leq C \xi^{\frac{r}{\lambda(r-1)+1}}$ implies that $\left.\phi^{-1}(\xi) \geq C \xi^{\lambda}\left(\phi_{*}^{-1}\right)\right)^{1-\lambda}$. Hence, by Lemma 2.1 and conservation of mass we derive that

$$
\|u\|_{L^{\phi}\left(\mathbb{R}^{d}\right)} \leq C\|u\|_{L^{r}\left(\mathbb{R}^{d}\right)}^{1-\lambda}
$$

and so (i) follows now from (3.4).

Let us prove (ii). We first note that if $\phi$ is of upper type $s$ then

$$
\|u\|_{L^{\phi}\left(\mathbb{R}^{d}\right)} \leq \begin{cases}C_{\phi}\left(\int_{\mathbb{R}^{d}} \phi(u)\right)^{1 / s} & \text { if }\|u\|_{L^{\phi}\left(\mathbb{R}^{d}\right)} \leq 1, \\ \int_{\mathbb{R}^{d}} \phi(u) & \text { if }\|u\|_{L^{\phi}\left(\mathbb{R}^{d}\right)}>1 .\end{cases}
$$

Indeed, if $\|u\|_{L^{\phi}\left(\mathbb{R}^{d}\right)}>1$ this is immediate from the convexity of $\phi$. On the other hand, if $\|u\|_{L^{\phi}\left(\mathbb{R}^{d}\right)} \leq 1$, we have

$$
1=\int_{\mathbb{R}^{d}} \phi\left(\frac{u}{\|u\|_{\phi}}\right) \leq \frac{C_{\phi}}{\|u\|_{\phi}^{s}} \int_{\mathbb{R}^{d}} \phi(u)
$$

and this proves (3.5).

Moreover, (ii) is a direct consequence of (3.3) and (3.5) and thus the corollary is proved.

Acknowledgements. Partially supported by CONICET and Secyt-UNC.

\section{REFERENCES}

[1] F. Andreu-Vaillo, J. M. Mazón, J. D. Rossi and J. J. Toledo-Melero. Nonlocal Diffusion Problems. Amer. Math. Soc. Mathematical Surveys and Monographs 2010. Vol. 165.

[2] F. Andreu, J. Mazón, J. D. Rossi, J. Toledo, A nonlocal p-Laplacian evolution equation with Neumann boundary conditions, J. Math. Pures Appl. 90 (2008), 201-227.

[3] F. Andreu, J. Mazón, J. D. Rossi, J. Toledo, The limit as $p \rightarrow \infty$ in a nonlocal p-Laplacian evolution equation: a nonlocal approximation of a model for sandpiles, Calc. Var. Partial Differential Equations. 35 (2009), 279-316.

[4] P. Bates, X. Chen, A. Chmaj, Heteroclinic solutions of a van der Waals model with indefinite nonlocal interactions, Calc. Var. Partial Differential Equations. 24 (2005), 261-281.

[5] E. Chasseigne, M. Chaves, J.D. Rossi, Asymptotic behavior for nonlocal diffusion equations, J. Math. Pures Appl. 86 (2006), 271-291.

[6] E. Di Nezza, G. Palatucci and E. Valdinoci, Hitchhiker's guide to the fractional Sobolev spaces, Bull. Sci. math., 136 (2012), No. 5, 521-573.

[7] S. Dipierro, X. Ros-Oton, and E. Valdinoci, Nonlocal problems with Neumann boundary conditions, (2014), eprint arXiv:1407.3313.

[8] Q. Du, M. Gunzburger, R. Lehoucq and K. Zhou. A nonlocal vector calculus, nonlocal volumeconstrained problems, and nonlocal balance laws. Math. Models Methods Appl. Sci. 23, (2013), 493-540.

[9] P. Fife. Some nonclassical trends in parabolic and parabolic-like evolutions, in "Trends in nonlinear analysis", pp. 153-191, Springer-Verlag, Berlin, 2003.

[10] E. Harboure, O. Salinas, B. Viviani, Relations between weighted Orlicz and $\mathrm{BMO}_{\varphi}$ spaces through fractional integrals, Comment. Math. Univ. Carolin. 40 (1999), 53-69.

[11] V. Hutson, S. Martínez, K. Mischaikow and G. T. Vickers. The evolution of dispersal, J. Math. Biol. 47 (2003), 483-517. 
[12] L. Ignat, J. D. Rossi, Decay estimates for nonlocal problems via energy methods, J. Math. Pures Appl. 92 (2009), 163-187.

[13] R. O'Neil, Fractional integration in Orlicz spaces. I, Trans Amer. Math. Soc. 115 (1965), 300-328.

[14] M. L. Parks, R. Lehoucq, S. Plimpton and S. Silling. Implementing peridynamics within a molecular dynamics code, Computer Physics Comm., 179, (2008), 777-783.

[15] S. A. Silling. Reformulation of Elasticity Theory for Discontinuities and Long-Range Forces. J. Mech. Phys. Solids, 48, (2000), 175-209.

[16] S. A. Silling and R. Lehoucq. Convergence of Peridynamics to Classical Elasticity Theory. J. Elasticity, 93 (2008), 13-37.

(J. D. Rossi)

COnicet and Depto. de Matemática, FCEyn, Universidad de Buenos Aires, Ciudad Universitaria, Pab 1 (1428), Buenos Aires, Argentina.

EMAIL: jrossi@dm.uba.ar

(U. Kaufmann and R. Vidal)

Famaf, Universidad Nacional de Cordoba, (5000), Cordoba, Argentina.

EMAIL: kaufmann@mate.uncor.edu, vidal@mate.uncor.edu 